\title{
THE STEFAN PROBLEM AND CONCAVITY
}

\author{
ALBERT CHAU AND BEN WEINKOVE
}

\begin{abstract}
We construct examples for the one-phase Stefan problem which show that $\alpha$-concavity of the solution is in general not preserved in time, for $0 \leq \alpha<1 / 2$. In particular, this shows that, in contrast to the case of the heat equation for a fixed convex domain, log concavity is not preserved for solutions of the Stefan problem.
\end{abstract}

\section{INTRODUCTION}

The one-phase Stefan problem is a free boundary problem used to model phase transitions in matter where the phase boundary moves with time. The initial data is given by a domain $\Omega_{0}$ in $\mathbb{R}^{n}$ and a function $u_{0}$ defined on its closure $\bar{\Omega}_{0}$ which vanishes on the boundary $\partial \Omega_{0}$ and is positive in the interior $\Omega_{0}$. For positive time $t$, the solution of the Stefan problem is a family of domains $\Omega_{t}$ and a function $u$, positive on $\Omega_{t}$, such that

$$
\begin{gathered}
u_{t}=\Delta u \text { in } \Omega_{t} \\
u=0 \text { on } \partial \Omega_{t},
\end{gathered}
$$

and that the Stefan boundary condition holds, which states that $\partial \Omega_{t}$ moves in the direction of the outward normal with speed $|\nabla u|$. This can be stated as follows: if $X(t)$ is a path in $\partial \Omega_{t} \subset \mathbb{R}^{n}$ whose derivative is normal to $\partial \Omega_{t}$ at $X(t)$ then

$$
\dot{X}(t)=-\nabla u(X(t), t) .
$$

The study of the Stefan problem has a long history, and we refer the reader to [3, 4, 11, 12, 14, 18, 19, 20, 21, 22, 23, 24 and the references therein for the basic existence and uniqueness results.

In a classic paper, Brascamp-Lieb [2] showed that log concavity is preserved along the heat equation on convex domains (see also [1, 5, 6, 7, 10, 15, 16, 17] for some related results). Daskalopoulos-Hamilton-Lee [8] showed that root concavity $(1 / 2$-concavity in the terminology below) is preserved for the porous medium equation, a degenerate diffusion equation. It is natural then to ask what if any concavity conditions are preserved for the Stefan problem [9].

Our main result is a negative one. We show that $\alpha$-concavity of the initial data, for $\alpha \in[0,1 / 2)$, is not in general preserved in time for the Stefan problem. Log concavity corresponds to $\alpha=0$ and so our result implies in particular that log concavity is not preserved.

More precisely, we will now define what we mean by a solution of the Stefan problem in the special case that it is of interest to us. Fix $k \geq 2$. Let $\Omega_{0}$ be a bounded domain and let $u_{0}$ be a function in $C^{k}\left(\bar{\Omega}_{0}\right)$ which vanishes on $\partial \Omega_{0}$, is strictly positive on $\Omega_{0}$ and whose derivative $\nabla u_{0}$ is nowhere vanishing on $\partial \Omega_{0}$. We define a nondegenerate $C^{k}$ solution of the (one-phase) Stefan problem starting with the initial data $\left(\Omega_{0}, u_{0}\right)$ on the time interval $[0, T]$ to be a relatively open set $\Omega \subset \mathbb{R}^{n} \times[0, T]$ and a function $u \in C^{k}(\bar{\Omega})$ satisfying the following conditions. For each $t \in[0, T]$, the set $\Omega_{t}:=\Omega \cap\left(\mathbb{R}^{n} \times\{t\}\right)$ is a 
bounded domain in $\mathbb{R}^{n} \times\{t\} \cong \mathbb{R}^{n}$ with $t=0$ corresponding to the initial domain $\Omega_{0}$. The function $\left.u\right|_{\Omega_{0}}$ is equal to the initial data $u_{0}$. The function $u$ is positive on $\Omega$ and vanishes on $\partial \Omega$, and $u$ satisfies (1.1) and (1.2). Moreover, $u$ satisfies the nondegeneracy condition that its spatial gradient $\nabla u$ does not vanish anywhere on $\partial \Omega$.

Some remarks are in order:

(1) In the above we use the parabolic definition of $C^{k}$, so that $u \in C^{k}$ means that $u$ has $k$ derivatives in the spatial directions and $k / 2$ derivatives in the time direction.

(2) When we refer to the boundary $\partial \Omega=\bar{\Omega} \backslash \Omega$, we are using the subspace topology on $\mathbb{R}^{n} \times[0, T]$. In particular $\Omega$ includes $\Omega_{0}$ and $\Omega_{T}$ while $\partial \Omega$ does not. By the nondegeneracy condition on $u$, the boundary $\partial \Omega$ inherits regularity from $u$.

(3) Our definition of a solution to the Stefan problem is rather restrictive since it insists that $u$ be $C^{k}$ at time $t=0$ in both space and time directions, up to the boundary. This imposes compatibility conditions on $u_{0}$ which are described in Section 2 below.

We now define $\alpha$-concavity in our setting. Let $W \subset \mathbb{R}^{n}$ be a bounded domain and let $v \in C^{2}(\bar{W})$ be positive on $W$ and vanishing on $\partial W$. For $\alpha>0$, we say that $v$ is $\alpha$-concave on $W$ if $D^{2} v^{\alpha} \leq 0$ on $W$. We say that $v$ is 0 -concave if $D^{2} \log v \leq 0$ on $W$. Equivalently, $\alpha$-concavity corresponds to the nonpositivity of the matrix with $(i, j)$ th entry

$$
\left(v D_{i} D_{j} v-(1-\alpha) D_{i} v D_{j} v\right)
$$

on $W$.

Our main theorem gives a family of examples in $\mathbb{R}^{2}$ for the Stefan problem which break the $\alpha$-concavity for positive time.

Theorem 1.1. Given $k \geq 2$ and $\alpha \in[0,1 / 2)$, there exist a bounded convex set $\Omega_{0} \subset \mathbb{R}^{2}$ with smooth boundary and $u_{0} \in C^{\infty}\left(\bar{\Omega}_{0}\right)$ which is strictly positive on $\Omega_{0}$ and vanishes on $\partial \Omega_{0}$ with the following properties:

(i) $u_{0}$ is $\alpha$-concave on $\Omega_{0}$.

(ii) $\nabla u_{0}$ does not vanish at any point of $\partial \Omega_{0}$.

(iii) There exists $T>0$ and a unique nondegenerate $C^{k}$ solution $(\Omega, u)$ of the Stefan problem starting at the initial data $\left(\Omega_{0}, u_{0}\right)$ on the time interval $[0, T]$ such that:

$$
\Omega_{t} \text { is not convex for any } t \in(0, T]
$$

and

$$
\left.u\right|_{\Omega_{t}} \text { is not } \alpha \text {-concave for any } t \in(0, T] .
$$

Our result in particular implies that additional assumptions are needed for Theorem 1.1 of $[9]$.

The outline of the paper is as follows. In Section 2 we give an overview of the compatibility conditions required for short time existence of $C^{k}$ solutions to the Stefan problem (in the sense described above). We also give some elementary results about concave functions, including their short proofs. Section 3 is the main part of the paper, which gives the construction of $\Omega_{0}$ and $u_{0}$. The proof of Theorem 1.1 is completed in Section 4 , 


\section{Preliminaries}

2.1. Short time existence result and compatibility conditions. The Stefan boundary condition (1.2) together with (1.1) induces compatibility conditions for $u_{0}$ on the boundary $\partial \Omega_{t}$. These arise from differentiating with respect to $t$ the equation

$$
u(X(t), t)=0
$$

along a path $X(t) \in \partial \Omega_{t}$ whose derivative is normal to the boundary, while using (1.2) and (1.1) to replace time derivatives with spatial derivatives. We illistrate this by deriving the first two compatibility conditions in detail as follows.

Differentiating (2.1) once in time gives $u_{t}+\nabla u \cdot \dot{X}=0$, then using (1.1) and (1.2) gives the first compatibility condition for $u_{0}$ :

$$
\Delta u_{0}-\left|\nabla u_{0}\right|^{2}=0 \text { on } \partial \Omega_{0} .
$$

Differentiating (2.1) once more in time gives $\Delta u_{t}+\nabla \Delta u \cdot \dot{X}-2 \nabla u_{t} \nabla u-2 u_{i} u_{i j} \dot{X}_{j}=0$, and using (1.1) and (1.2) we obtain the second compatibility condition for $u_{0}$ :

$$
\Delta^{2} u_{0}-3 \nabla \Delta u_{0} \cdot \nabla u_{0}+2 \sum_{i, j}\left(u_{0}\right)_{i j}\left(u_{0}\right)_{i}\left(u_{0}\right)_{j}=0 \text { on } \partial \Omega_{0} .
$$

In general, we see that the $k$ th compatibility condition for the initial condition for $u_{0}$ can be written as

$$
\Delta^{k}\left(u_{0}\right)+F_{k}\left(u_{0}\right)=0 \text { on } \partial \Omega_{0},
$$

where $F_{k}$ is a differential operator of degree at most $2 k-1$ and is obtained as above, namely by differentating (2.1) $k$ times in $t$, then using (1.1) and (1.2) to replace time derivatives of $u_{0}$ with spatial derivatives.

A result of Hanzawa [14] (see also [21]) states that solutions to the Stefan problem exist on a small time interval $[0, T]$ as long as $u_{0}$ satisfies compatibility conditions. For our purposes we may assume that we are given the initial data of a smooth function $u_{0} \in C^{\infty}\left(\bar{\Omega}_{0}\right)$ where $\Omega_{0}$ is a bounded domain whose boundary $\partial \Omega_{0}$ is smooth and has only one component. The function $u_{0}$ is strictly positive on $\Omega_{0}$, vanishes on $\partial \Omega_{0}$ and its derivative $\nabla u_{0}$ is nowhere vanishing on $\partial \Omega_{0}$. In this setting, Hanzawa's result can be stated as follows.

Theorem 2.1. Fix $k \geq 2$. Then there exists $N=N(k)$ such that if $u_{0}$ satisfies the first $N$ compatibility conditions then there exists $T>0$ and a unique nondegenerate $C^{k}$ solution of the Stefan problem $(\Omega, u)$ on the time interval $[0, T]$ starting with this initial data.

The constant $N=N(k)$ is given explicitly in [14 and is not optimal, but here we are not concerned with the question of optimal regularity.

2.2. Two elementary lemmas about concave functions. In this section we recall two known, elementary results which will be needed in the sequel. Let $r$ denote the distance from the origin in $\mathbb{R}^{2}$. 
Lemma 2.1. Let $q(r)$ be a $C^{2}$ radial function on $\mathbb{R}^{2}$. Then $q$ is strongly concave on the set

$$
S=\left\{(x, y) \in \mathbb{R}^{2} \mid r \neq 0, q^{\prime}(r)<0, \text { and } q^{\prime \prime}(r)<0\right\} .
$$

Proof. This is a straightforward computation, using the fact that $r_{x}=x / r$ and $r_{y}=y / r$. At a point in $S$ we have

$$
\begin{aligned}
& q_{x}=\frac{x}{r} q^{\prime}, \quad q_{x x}=\frac{x^{2}}{r^{2}} q^{\prime \prime}+\frac{1}{r} q^{\prime}-\frac{x^{2}}{r^{3}} q^{\prime}=\frac{x^{2}}{r^{2}} q^{\prime \prime}+\frac{y^{2}}{r^{3}} q^{\prime}<0 \\
& q_{y}=\frac{y}{r} q^{\prime}, \quad q_{y y}=\frac{y^{2}}{r^{2}} q^{\prime \prime}+\frac{1}{r} q^{\prime}-\frac{y^{2}}{r^{3}} q^{\prime}=\frac{y^{2}}{r^{2}} q^{\prime \prime}+\frac{x^{2}}{r^{3}} q^{\prime}<0 \\
& q_{x y}=\frac{x y}{r^{2}} q^{\prime \prime}-\frac{x y}{r^{3}} q^{\prime}, \quad q_{x x} q_{y y}-q_{x y}^{2}=\frac{1}{r} q^{\prime} q^{\prime \prime}>0
\end{aligned}
$$

as required.

Next we have the following elementary lemma about extending concave functions [13.

Lemma 2.2. Let $W$ be an open bounded convex set in $\mathbb{R}^{n}$ and let $f$ be a smooth realvalued function defined on the set

$$
W^{\delta}=\{p \in W \mid \operatorname{dist}(p, \partial W)<\delta\},
$$

for some $\delta>0$. Assume that there exists a constant $c \in[-\infty, \infty)$ such that $f(p)>c$ on $W^{\delta}$ and $f(p) \rightarrow c$ as $p$ tends to any point in $\partial W$. Also assume that $D^{2} f<0$ and $D f \neq 0$ on $W^{\delta}$. Then there exists a smooth concave function $F: W \rightarrow(c, \infty)$ which coincides with $f$ on $W^{\delta^{\prime}}$ for some $0<\delta^{\prime}<\delta$.

Moreover, if $W$ is a ball in $\mathbb{R}^{n}$ centered at a point $P$ and $f$ a function of the distance $r$ from $P$, then $F$ can also be taken to be a function of $r$.

Proof. The proof is essentially contained in [13], but we give the argument here for the sake of completeness. First note that the level sets $\{f=a\}$ for constants $a$ close to (and strictly larger than) $c$ are smooth convex hypersurfaces contained in $W^{\delta}$. If $c=-\infty$ then " $a$ close to $c$ " means that $a$ is sufficiently negative.

Fix now such an $a>c$. Define a function $\tilde{f}: W \rightarrow \mathbb{R}$ by

$$
\tilde{f}= \begin{cases}f, & \text { on }\{f \leq a\} \\ a, & \text { otherwise. }\end{cases}
$$

Then the function $\tilde{f}$ is locally concave on $W$ and hence concave on $W$ (away from $\{f=a\}$ it satisfies $D^{2} \tilde{f} \leq 0$ and near $\{f=a\}$ it is the minimum of two concave functions).

For small $\varepsilon>0$ let $\tilde{f}_{\varepsilon}=\tilde{f} * \eta_{\varepsilon}$ be the convolution of $\tilde{f}$ by a mollifier $\eta_{\varepsilon}$ given by $\eta_{\varepsilon}(p)=\varepsilon^{-n} \eta\left(\varepsilon^{-1} p\right)$ for $\eta$ a smooth nonnegative function supported in the unit ball $B$ with $\int_{B} \eta d x=1$. Then $\tilde{f}_{\varepsilon}$ is smooth and concave on its domain of definition $W_{\varepsilon}:=\{p \in W \mid \operatorname{dist}(p, \partial W)>\varepsilon\}$.

Now fix $a^{\prime}$ with $a>a^{\prime}>c$. Then $\tilde{f}$ is smooth and concave on $\left\{f<a^{\prime}\right\}$. Choose a smooth bump function $\phi$ which is equal to 0 on $\left\{f \geq a^{\prime}\right\}$ and is equal to 1 on $W^{\delta^{\prime}}$ for some small $\delta^{\prime}>0$. 
Define a smooth function $F: W \rightarrow \mathbb{R}$ by

$$
F=(1-\phi) \tilde{f}_{\varepsilon}+\phi \tilde{f} .
$$

Then $F$ agrees with the concave function $\tilde{f}$ and hence $f$ on $W^{\delta^{\prime}}$ and agrees with the concave function $\tilde{f}_{\varepsilon}$ on $\left\{f \geq a^{\prime}\right\}$. Here we choose $\varepsilon>0$ small enough so that $\tilde{f}_{\varepsilon}$ is defined on the complement of $W^{\delta^{\prime}}$. It remains to check that $F$ is concave on the compact set $K=\left\{f \leq a^{\prime}\right\} \backslash W^{\delta^{\prime}}$. Write $F=\tilde{f}_{\varepsilon}+\phi\left(\tilde{f}-\tilde{f}_{\varepsilon}\right)$. Then

$$
F_{i j}=\left(\tilde{f}_{\varepsilon}\right)_{i j}+\phi_{i j}\left(\tilde{f}-\tilde{f}_{\varepsilon}\right)+\phi_{i}\left(\tilde{f}-\tilde{f}_{\varepsilon}\right)_{j}+\phi_{j}\left(\tilde{f}-\tilde{f}_{\varepsilon}\right)_{i}+\phi\left(\tilde{f}-\tilde{f}_{\varepsilon}\right)_{i j} .
$$

The result now follows from the fact that $\tilde{f}_{\varepsilon} \rightarrow f$ uniformly $C^{2}$ on $K$ as $\varepsilon \rightarrow 0$. Indeed, since $D^{2} f<0$ on the compact set $K$, it is uniformly strongly concave on $K$, so we can choose $\varepsilon>0$ small enough that that the matrix $\left(\tilde{f}_{\varepsilon}\right)_{i j}$ is uniformly negative definite. But since $\tilde{f}_{\varepsilon} \rightarrow f$ uniformly $C^{2}$ on $K$ as $\varepsilon \rightarrow 0$, all the other terms tend to zero, so for $\varepsilon>0$ sufficiently small we get $\left(F_{i j}\right)<0$ on $K$.

Finally, in the case when $W$ is a ball in $\mathbb{R}^{n}$ centered at a point $P$ and $f$ is a function of the distance $r$ from $P$, we define $\tilde{f}_{\varepsilon}(r)$ to be the convolution of $\tilde{f}(r)$ with a standard mollifier as a function of $r$. We also choose the bump function $\phi$ to be a function of $r$, and it follows that $F$ is a function of $r$.

\section{Construction of the initial data}

Our starting point is a radial function defined on a disk. Fix an integer $N \geq 1$ and some $\alpha \in[0,1 / 2)$ throughout the section. Let $D$ be the open disk in $\mathbb{R}^{2}$ of radius 2 centered at the point $(0,2)$, and let $r$ denote the distance from $(0,2)$.

Proposition 3.1. There exists a smooth function $U$ on the closed disk $\bar{D}$ satisfying

(a) $U$ is positive on $D$, vanishes on $\partial D$, and $\nabla U$ is nowhere zero on $\partial D$.

(b) $U$ is $\alpha$-concave on $D$.

(c) $U$ satisfies the compatibility conditions (2.4) on $\partial D$, for $k=1, \ldots, N$. Moreover, $U$ is a function of $r$.

Proof. Such a function $U(x, y)$ is easily constructed as follows. Writing $r$ for the distance from $(0,2)$, let $q:[0,2] \rightarrow \mathbb{R}$ be a smooth function satisfying

$$
q(r)>0,0 \leq r<2, q(2)=0
$$

and

$$
q^{\prime}(2)<0, q^{\prime \prime}(2)+\frac{1}{2} q^{\prime}(2)>0 .
$$

Then for $\alpha \in(0,1 / 2)$ we see that for $r$ close to 2 we have

$$
\left(q^{\alpha}\right)^{\prime}=\alpha q^{\alpha-1} q^{\prime}<0,
$$

and

$$
\left(q^{\alpha}\right)^{\prime \prime}=\alpha q^{\alpha-2}\left((\alpha-1)\left(q^{\prime}\right)^{2}+q q^{\prime \prime}\right)<0 .
$$

For the case $\alpha=0$ replace $q^{\alpha}$ by $\log q$ and the same holds. This implies that if we define $U(x, y)=q(r)$ for $r$ close to 2 then $U$ is strongly $\alpha$-concave there by Lemma 2.1. To satisfy $\Delta U=|\nabla U|^{2}$ on $\partial D$ we note that by (3.2), the quantities $\Delta U$ and $|\nabla U|^{2}$ are strictly positive on $\partial D$ and hence we can scale $U$ to ensure that the first compatibility condition holds. 
We can then recursively prescribe $q^{(2 k)}(2)$ for $k=2,3, \ldots, N$ so that the $k$ th compatibility condition (2.4) for $U$ holds up to order $N$. This does not affect the positivity or $\alpha$-concavity of $U$ near $\partial D$. We can then extend $U$ by Lemma 2.2 to a positive $\alpha$-concave function inside $D$.

We now define a new convex domain $\Omega_{0}$, obtained by modifying the disk $D$. The part of the boundary $\partial D$ below the line $y=2$ can be written as a graph $y=G(x)$ for

$$
G(x)=2-\sqrt{4-x^{2}}, \quad-2<x<2 .
$$

Let $\delta \in(0,1 / 4)$ be a small positive constant depending only on $\alpha$, to be determined later. Define a new function $g:(-2,2) \rightarrow \mathbb{R}$ by modifying $G$ as follows:

$$
g(x)= \begin{cases}1 / 20, & x \in[-\delta, \delta] \\ G(x), & x \in(-2,-1 / 2] \cup[1 / 2,2)\end{cases}
$$

and extend $g$ to be a smooth function on $(-2,2)$ so that $g^{\prime \prime}>0$ on the remaining intervals $(-1 / 2,-\delta)$ and $(\delta, 1 / 2)$. Note that $g$ is a convex function. We now define our convex domain to be

$$
\Omega_{0}=D \cap\{(x, y) \in(-2,2) \times \mathbb{R} \mid y>g(x)\} .
$$

Note that $\partial \Omega_{0}$ is smooth, and contains a line segment $[-\delta, \delta] \times\{1 / 20\}$.

The main result of this paper is the following construction:

Theorem 3.1. There is a smooth function $v$ on $\bar{\Omega}_{0}$ satisfying the following conditions:

(a) $v$ is positive on $\Omega_{0}$, vanishes on $\partial \Omega_{0}$.

(b) $v$ is $\alpha$-concave on $\Omega_{0}$.

(c) $\nabla v$ does not vanish at any point of $\partial \Omega_{0}$.

(d) $v$ satisfies the compatibility conditions (2.4) on $\partial \Omega_{0}$, for $k=1, \ldots, N$.

(e) The map

$$
x \mapsto v_{y}(x, 1 / 20), \quad \text { for } x \in[-\delta, \delta],
$$

is positive and strongly convex (namely $\left(v_{y}\right)_{x x}>0$ ).

Proof. We obtain $v$ by modifying the function $U$ constructed in Proposition 3.1, which we may assume for convenience is defined as a smooth function on all of $\mathbb{R}^{2}$. We may write $U(x, y)$ on $[-1,1] \times[-1,1]$ as

$$
U(x, y)=\sum_{\ell=1}^{2 N}(y-G(x))^{\ell} E_{\ell}(x)+(y-G(x))^{2 N+1} R(x, y)
$$

for smooth functions $E_{1}, \ldots, E_{2 N}, R$, where we recall that $G$ is defined by (3.3). Indeed, this follows by considering the new variable $\tilde{y}=y-G(x)$, and considering for each $x \in[-1,1]$ the Taylor expansion of the function $U(x, \tilde{y})$ in powers of $\tilde{y}$ around $\tilde{y}=0$ and using the Taylor remainder theorem.

We now define a function $v(x, y)$ on $[-1,1] \times[-1,1]$ by

$$
v(x, y)=\sum_{\ell=1}^{2 N}(y-g(x))^{\ell} e_{\ell}(x)+(y-g(x))^{2 N+1} R(x, y)
$$

where $g(x)$ is as in (3.4) and the smooth functions $e_{1}, \ldots, e_{2 N}$ are to be determined. 
For simplicity of notation, in what follows we will denote $E_{1}, E_{2}$ by $F, H$ and $e_{1}, e_{2}$ by $f, h$ respectively. Note that since $\nabla U$ is nonvanishing on $\partial D$, there exists a constant $c>0$ such that

$$
F(x) \geq c>0 .
$$

We define $f$ as follows. First choose $\delta \in(0,1 / 4)$ so that the solution $\psi(x)$ of the ODE problem

$$
\begin{gathered}
\psi \psi^{\prime \prime}-\frac{2}{3}(1-2 \alpha)\left(\psi^{\prime}\right)^{2}=-1, \quad \text { on }[-2 \delta, 2 \delta], \\
\psi(0)=1, \psi^{\prime}(0)=\sqrt{2 /(1-2 \alpha)}
\end{gathered}
$$

satisfies $\psi^{\prime \prime}>0$ and $\psi>0$ on $[-2 \delta, 2 \delta]$. Then we define $f$ to be a smooth function on $[-1,1]$ such that

$$
f(x)= \begin{cases}\psi(x), & \text { for }-2 \delta \leq x \leq 2 \delta \\ F(x), & \text { for } x \in[-1,-1 / 2] \cup[1 / 2,1]\end{cases}
$$

and

$$
f(x) \geq c>0 \quad \text { on }[-1,1]
$$

where we assume without loss of generality that $c$ is the same constant as in (3.6). Observe that from the definition of $f$ and $g$, we have $v_{y}(x, 1 / 20)=\psi(x)$ for $x \in[-\delta, \delta]$ and hence $v(x, y)$ will satisfy (e).

We are interested only in the behavior of $v$ close to the boundary $y=g(x)$ and so in what follows we can neglect some higher order terms of $|y-g|$. Write

$$
v=(y-g) f+(y-g)^{2} h+O\left(|y-g|^{3}\right)
$$

Differentiating this we obtain

$$
\begin{aligned}
v_{y}= & f+2(y-g) h+O\left(|y-g|^{2}\right) \\
v_{x}= & -g^{\prime} f+(y-g) f^{\prime}-2(y-g) g^{\prime} h+(y-g)^{2} h^{\prime} \\
& +(y-g)^{2} O\left(\left|g^{\prime}\right|\right)+O\left(|y-g|^{3}\right) \\
v_{y y}= & 2 h+O(|y-g|) \\
v_{x y}= & f^{\prime}-2 g^{\prime} h+2(y-g) h^{\prime}+(y-g) O\left(\left|g^{\prime}\right|\right)+O\left(|y-g|^{2}\right) \\
v_{x x}= & -g^{\prime \prime} f-2 g^{\prime} f^{\prime}+2\left(g^{\prime}\right)^{2} h+(y-g) f^{\prime \prime}+(y-g) O\left(\left|g^{\prime}\right|,\left|g^{\prime \prime}\right|\right)+O\left(|y-g|^{2}\right) .
\end{aligned}
$$

We will now choose the function $h(x)$ so that $v$ satisfies the first compatibility condition $\Delta v=|\nabla v|^{2}$ on the boundary $\{y=g(x)\}$. Observe that on $(x, g(x))$ for $x \in[-1,1]$,

$$
\Delta v=2 h-g^{\prime \prime} f-2 g^{\prime} f^{\prime}+2\left(g^{\prime}\right)^{2} h
$$

and

so that we require

$$
|\nabla v|^{2}=v_{x}^{2}+v_{y}^{2}=\left(g^{\prime}\right)^{2} f^{2}+f^{2},
$$

$$
h(x)=\frac{\left(g^{\prime}\right)^{2} f^{2}+f^{2}+g^{\prime \prime} f+2 g^{\prime} f^{\prime}}{2\left(1+\left(g^{\prime}\right)^{2}\right)} .
$$

We define $h(x)$ by (3.9). It follows that $v(x, y)$ satisfies the first compatibility condition $\Delta v=|\nabla v|^{2}$ on $\partial \Omega_{0} \bigcap[-1,1] \times[-1,1]$. Moreover, since our function $U$ constructed in 
Proposition 3.1 satisfies the first compatibility condition $\Delta U=|\nabla U|^{2}$ on the boundary, we have

$$
H(x)=\frac{\left(G^{\prime}\right)^{2} F^{2}+F^{2}+G^{\prime \prime} F+2 G^{\prime} F^{\prime}}{2\left(1+\left(G^{\prime}\right)^{2}\right)},
$$

for $x \in[-1,1]$. Since $f=F$ and $g=G$ whenever $x$ is in the complement of $[-1 / 2,1 / 2]$, it follows that $h=H$ in the complement of $[-1 / 2,1 / 2]$.

We can now similarly define $e_{3}, e_{4}, \ldots, e_{2 N}$ so that $v(x, y)$ satisfies the $k$ th compatibility conditions for $k=2,3, \ldots, N$. Indeed applying the operator $\Delta^{2}$ to (3.5) and evaluating on $y=g(x)$, the second compatibility condition takes the form

$$
\begin{aligned}
e_{4}(x)= & \text { smooth expression in terms of } e_{3}(x), g(x), f(x), h(x) \\
& \text { and their derivatives. }
\end{aligned}
$$

We can simply define $e_{3}(x)=E_{3}(x)$. Define $e_{4}(x)$ by the formula (3.10) so that $v(x, y)$ satisfies the second compatibility condition. Then since $U$ satisfies the second compatibility condition, and $f=F, g=G, h=H, e_{3}=E_{3}$ when $x$ is in the complement of $[-1 / 2,1 / 2]$ it follows that $e_{4}=E_{4}$ in the complement of $[-1 / 2,1 / 2]$.

Continuing inductively, we define $e_{5}, \ldots, e_{2 N}$ so that the $k$ th compatibility conditions are satisfied for $k=2,3, \ldots, N$. Moreover when $x$ is in the complement of $[-1 / 2,1 / 2]$, the functions $f, g, h, e_{3}, \ldots, e_{2 N}$ coincide with $F, G, H, E_{3}, \ldots, E_{2 N}$ respectively.

Finally we will show that $v$ is $\alpha$-concave in the set

$$
S_{\varepsilon}=\{(x, y) \mid-1 \leq x \leq 1, g(x)<y<g(x)+\varepsilon\},
$$

for $\varepsilon>0$ sufficiently small.

For this, we need to show that for all $x \in[-1,1]$ and $y-g(x)$ sufficiently small and positive we have

$$
v v_{x x}-(1-\alpha) v_{x}^{2}<0, v v_{y y}-(1-\alpha) v_{y}^{2}<0
$$

and

$$
\left(v v_{x x}-(1-\alpha) v_{x}^{2}\right)\left(v v_{y y}-(1-\alpha) v_{y}^{2}\right)-\left(v v_{x y}-(1-\alpha) v_{x} v_{y}\right)^{2}>0 .
$$

We compute the terms above separately using (3.8),

$$
\begin{aligned}
& v v_{x x}-(1-\alpha) v_{x}^{2} \\
= & \left((y-g) f+(y-g)^{2} h\right)\left(-g^{\prime \prime} f-2 g^{\prime} f^{\prime}+2\left(g^{\prime}\right)^{2} h+(y-g) f^{\prime \prime}\right) \\
& -(1-\alpha)\left(-g^{\prime} f+(y-g) f^{\prime}-2(y-g) g^{\prime} h+(y-g)^{2} h^{\prime}\right)^{2} \\
& +(y-g)^{2} O\left(\left|g^{\prime}\right|,\left|g^{\prime \prime}\right|\right)+O\left(|y-g|^{3}\right) \\
= & -(1-\alpha)\left(g^{\prime}\right)^{2} f^{2}+(y-g)\left(-g^{\prime \prime} f^{2}-2 \alpha f f^{\prime} g^{\prime}-2(1-2 \alpha) f\left(g^{\prime}\right)^{2} h\right) \\
& +(y-g)^{2}\left(f f^{\prime \prime}-(1-\alpha)\left(f^{\prime}\right)^{2}+O\left(\left|g^{\prime}\right|,\left|g^{\prime \prime}\right|\right)\right), \\
& +O\left(|y-g|^{3}\right) .
\end{aligned}
$$

Observe that the zero order term in $(y-g)$ is negative, as is the first order term $-(y-g) g^{\prime \prime} f^{2}$. To deal with the term $-2 \alpha(y-g) f f^{\prime} g^{\prime}$ we argue as follows. If $\alpha \neq 0$, 
define

$$
\eta=\frac{1-4 \alpha^{2}}{1-\alpha^{2}} \in(0,1)
$$

and use the inequality

$$
-2 \alpha(y-g) f f^{\prime} g^{\prime} \leq(1-\eta)(1-\alpha)\left(g^{\prime}\right)^{2} f^{2}+(y-g)^{2} \frac{\alpha^{2}}{(1-\alpha)(1-\eta)}\left(f^{\prime}\right)^{2}
$$

to obtain on $S_{\varepsilon}$, for $\varepsilon>0$ sufficiently small,

$$
\begin{aligned}
v v_{x x}-(1-\alpha) v_{x}^{2} \leq & -\eta(1-\alpha)\left(g^{\prime}\right)^{2} f^{2}-2(y-g)(1-2 \alpha) f\left(g^{\prime}\right)^{2} h \\
& +(y-g)^{2}\left(f f^{\prime \prime}-\left\{(1-\alpha)-\frac{\alpha^{2}}{(1-\alpha)(1-\eta)}\right\}\left(f^{\prime}\right)^{2}+O\left(\left|g^{\prime}\right|,\left|g^{\prime \prime}\right|\right)\right) \\
& +O\left(|y-g|^{3}\right) \\
\leq & -\frac{\eta}{2}(1-\alpha)\left(g^{\prime}\right)^{2} f^{2}+(y-g)^{2}\left(f f^{\prime \prime}-\frac{2}{3}(1-2 \alpha)\left(f^{\prime}\right)^{2}+O\left(\left|g^{\prime}\right|,\left|g^{\prime \prime}\right|\right)\right), \\
& +O\left(|y-g|^{3}\right),
\end{aligned}
$$

where have absorbed the first order term in the zero and second order terms, using the fact that $f \geq c>0$ and $h$ is bounded. This inequality holds for $\alpha=0$ too, taking $\eta=1$. By definitions of $f$ and $g$, there exists a small constant $\rho>0$ such that if $-\delta-\rho \leq x \leq \delta+\rho$ then

$$
f f^{\prime \prime}-\frac{2}{3}(1-2 \alpha)\left(f^{\prime}\right)^{2}=-1
$$

and $g^{\prime}, g^{\prime \prime}$ is sufficiently small so that the second order term is strictly negative. Otherwise $\left(g^{\prime}\right)^{2}$ is uniformly positive and so the zero order term dominates. In either case there exists a uniform $a>0$ such that on $S_{\varepsilon}$,

$$
v v_{x x}-(1-\alpha) v_{x}^{2} \leq-a(y-g)^{2}
$$

as long as $\varepsilon>0$ is sufficiently small.

Next we compute on $S_{\varepsilon}$,

$$
\begin{aligned}
v v_{y y}-(1-\alpha) v_{y}^{2}= & \left((y-g) f+(y-g)^{2} h\right) 2 h \\
& -(1-\alpha)(f+2(y-g) h)^{2}+O\left(|y-g|^{2}\right) \\
= & -(1-\alpha) f^{2}-2(y-g)(1-2 \alpha) f h+O\left(|y-g|^{2}\right) \\
\leq & -a<0,
\end{aligned}
$$

for a uniform $a>0$ uniform as long as $\varepsilon>0$ is sufficiently small, using the lower bound on $f$ of (3.7). 
Next we compute

$$
\begin{aligned}
& v v_{x y}-(1-\alpha) v_{x} v_{y} \\
= & \left((y-g) f+(y-g)^{2} h\right)\left(f^{\prime}-2 g^{\prime} h+2(y-g) h^{\prime}\right) \\
& -(1-\alpha)\left(-g^{\prime} f+(y-g) f^{\prime}-2(y-g) g^{\prime} h+(y-g)^{2} h^{\prime}\right)(f+2(y-g) h) \\
& +(y-g)^{2} O\left(\left|g^{\prime}\right|\right)+O\left(|y-g|^{3}\right) \\
= & (1-\alpha) g^{\prime} f^{2}+(y-g)\left(\alpha f f^{\prime}+2(1-2 \alpha) f g^{\prime} h\right) \\
& +(y-g)^{2}\left(-(1-2 \alpha) h f^{\prime}+(1+\alpha) f h^{\prime}+O\left(\left|g^{\prime}\right|\right)\right)+O\left(|y-g|^{3}\right) .
\end{aligned}
$$

Combining with (3.11) and (3.13), we finally obtain

$$
\begin{aligned}
& \left(v v_{x x}-(1-\alpha) v_{x}^{2}\right)\left(v v_{y y}-(1-\alpha) v_{y}^{2}\right)-\left(v v_{x y}-(1-\alpha) v_{x} v_{y}\right)^{2} \\
= & (1-\alpha)(y-g) g^{\prime \prime} f^{4} \\
& +(y-g)^{2}\left(-(1-\alpha) f^{2}\left(f f^{\prime \prime}-(1-\alpha)\left(f^{\prime}\right)^{2}\right)-\alpha^{2} f^{2}\left(f^{\prime}\right)^{2}+O\left(\left|g^{\prime}\right|,\left|g^{\prime \prime}\right|\right)\right) \\
& +O\left(|y-g|^{3}\right) \\
\geq & a|y-g|^{2},
\end{aligned}
$$

for some uniform $a>0$ uniform as long as $\varepsilon>0$ is sufficiently small. To see this inequality we argue as follows. Note that the coefficient of $(y-g)$ is nonnegative. There exists a small $\rho>0$ such that if $-\delta-\rho \leq x \leq \delta+\rho$ then $f f^{\prime \prime}-\frac{2}{3}(1-2 \alpha)\left(f^{\prime}\right)^{2}=-1$ and $g^{\prime}, g^{\prime \prime}$ is sufficiently small so that for these values of $x$,

$$
\begin{aligned}
& -(1-\alpha) f^{2}\left(f f^{\prime \prime}-(1-\alpha)\left(f^{\prime}\right)^{2}\right)-\alpha^{2} f^{2}\left(f^{\prime}\right)^{2}+O\left(\left|g^{\prime}\right|,\left|g^{\prime \prime}\right|\right) \\
= & -(1-\alpha) f^{2}\left(f f^{\prime \prime}-\frac{1-2 \alpha}{1-\alpha}\left(f^{\prime}\right)^{2}\right)+O\left(\left|g^{\prime}\right|,\left|g^{\prime \prime}\right|\right) \\
\geq & -(1-\alpha) f^{2}\left(f f^{\prime \prime}-\frac{2}{3}(1-2 \alpha)\left(f^{\prime}\right)^{2}\right)+O\left(\left|g^{\prime}\right|,\left|g^{\prime \prime}\right|\right) \\
= & (1-\alpha) f^{2}+O\left(\left|g^{\prime}\right|,\left|g^{\prime \prime}\right|\right)>a>0,
\end{aligned}
$$

using the fact that $1 /(1-\alpha) \geq 1 \geq 2 / 3$. For $x$ not in this range, $g^{\prime \prime}$ is uniformly positive and so the first order term in $(y-g)$ dominates. This establishes (3.14).

Combining (3.12), (3.13) and (3.14) we see that $v$ is $\alpha$-concave on $S_{\varepsilon}$ for $\varepsilon>0$ sufficiently small. Moreover, $v(x, y)$ agrees with the function $U(x, y)$ for $x$ in the complement of $[-1 / 2,1 / 2]$ and hence we can extend $v$ (by simply setting equal to $U(x, y)$ for $(x, y) \notin[-1,1] \times[-1,1])$ to give a smooth $\alpha$-concave function, still referred to as $v$, on the set

$$
\Omega_{0}^{\varepsilon}=\left\{p \in \Omega_{0} \mid \operatorname{dist}\left(p, \partial \Omega_{0}\right)<\varepsilon\right\} .
$$

Moreover $v$ has nonvanishing derivative on the boundary $\partial \Omega_{0}$. Applying Lemma 2.2 completes the proof of the theorem. 


\section{Convexity Breaking}

In this section we complete the proof of Theorem 1.1.

Let $k \geq 2$ be given, and choose $N=N(k)$ sufficiently large as in Theorem 2.1. We take our initial data $u_{0}$ to be the function $v \in C^{\infty}\left(\bar{\Omega}_{0}\right)$ constructed in Theorem 3.1 for this given $N$ so that by Theorem 2.1 we have a nondegenerate $C^{k}$ solution $(\Omega, u)$ of the Stefan problem with this initial data on $[0, T]$. Let $p$ be the point $(0,1 / 20) \in \mathbb{R}^{2}$ and consider the disc $D_{\delta / 2}(p)$ of radius $\delta / 2$ centered at $p$. Shrinking $T$ if necessary, the solution $u$ has nonvanishing derivative on the boundary $\partial \Omega_{t}$. Hence by the Implicit Function Theorem, the free boundary $\partial \Omega_{t} \cap D_{\delta / 2}(p)$ is given by a graph $y=w(x, t)$ for a locally defined $C^{k}$ function $w$. Moreover, $w(x, 0)=1 / 20$ and by the Stefan boundary condition (1.2) the function $w_{t}(x, 0)$ coincides with $-v_{y}(x, 1 / 20)$ and so by part (e) of Theorem 3.1,

$$
x \mapsto w_{t}(x, 0)
$$

is strictly negative and strongly concave. Here, and in what follows, we may increase $k$ without loss of generality as necessary.

To show that $\Omega_{t}$ is not convex it is sufficient to show that for $t \in(0, T]$ the function $w(x, t)$ is not convex as a function of $x$. This however is an immediate consequence of Taylor's Theorem which gives

$$
w(x, t)=1 / 20+w_{t}(x, 0) t+R(x, t) t
$$

for $x \in[-\delta / 2, \delta / 2]$ and $t \in[0, T]$, where

$$
R(x, t)=\int_{0}^{t} w_{t t}(x, s) \frac{(t-s)}{t} d s .
$$

As $t \rightarrow 0$, the remainder term $R(x, t)$ tends to zero in the $C^{2}$ norm with respect to $x$. Hence, shrinking $T$ if necessary we obtain $w_{x x}(x, t)<0$ for $t \in(0, T]$ and in particular, $x \mapsto w(x, t)$ is not convex.

This immediately implies that $\left.u\right|_{\Omega_{t}}$ is not $\alpha$-concave for $t \in(0, T]$. Indeed, fix $t \in$ $(0, T]$, write $u=\left.u\right|_{\Omega_{t}}$ and consider the set $\{u(x, y)=\varepsilon\}$ for a small $\varepsilon>0$, near the point $(0,1 / 20)$. Shrinking $\varepsilon$ if necessary we may assume that $\nabla u$ does not vanish there and $\{u=\varepsilon\}$ is given locally by a $C^{2}$ graph $y=\rho(x)$ which has $\rho^{\prime \prime}(0)<0$. Rotating and translating the coordinates we may assume that $\rho(0)=\rho^{\prime}(0)=0$ and hence $u_{x}(0,0)=0$. Differentiating twice the equation $u(x, \rho(x))=\varepsilon$ gives $u_{x x}(0,0)=-u_{y}(0,0) \rho^{\prime \prime}(0)>0$. Hence $u u_{x x}-(1-\alpha) u_{x}^{2}>0$ at $(0,0)$ and so $u$ is not $\alpha$-concave.

This completes the proof of Theorem 1.1 .

\section{REFERENCES}

1. Borell, C., Brownian motion in a convex ring and quasiconcavity, Comm. Math. Phys. 86 (1982), no. 1, 143-147.

2. Brascamp, H.J., Lieb, E.H., On extensions of the Brunn-Minkowski and Prékopa-Leindler theorems, including inequalities for log concave functions, and with an application to the diffusion equation, J. Functional Analysis 22 (1976), no. 4, 366-389.

3. Caffarelli, L., Some aspects of the one-phase Stefan problem, Indiana Univ. Math. J. 27 (1978), no. $1,73-77$.

4. Caffarelli, L., Friedman, A., Continuity of the temperature in the Stefan problem, Indiana Univ. Math. J. 28 (1979), no. 1, 53-70. 
5. Chau, A., Weinkove, B., Counterexamples to quasiconcavity for the heat equation, to appear in Int. Math. Res. Not., arXiv:1802.04770.

6. Chau, A., Weinkove, B., Strong space-time convexity and the heat equation, preprint, arXiv:1903.03208

7. Chen, C.Q., Ma, X.-N., Salani, P., On space-time quasiconcave solutions of the heat equation, Mem. Amer. Math. Soc. 259 (2019), no. 1244.

8. Daskalopoulos, P., Hamilton, R., Lee, K., All time $C^{\infty}$-regularity of the interface in degenerate diffusion: a geometric approach, Duke Math. J. 108 (2001), no. 2, 295-327.

9. Daskalopoulos, P, Lee, K., All time smooth solutions of the one-phase Stefan problem and the HeleShaw flow, Comm. Partial Differential Equations 29 (2004), no. 1-2, 71-89.

10. Diaz, J.I., Kawohl, B., On convexity and starshapedness of level sets for some nonlinear elliptic and parabolic problems on convex rings, J. Math. Anal. Appl. 177 (1993), no. 1, 263-286.

11. Friedman, A., The Stefan problem in several space variables, Trans. Amer. Math. Soc. 133 (1968), 51-87.

12. Friedman, A., Kinderlehrer, D., A one phase Stefan problem, Indiana Univ. Math. J. 24 (1974/75), no. 11, 1005-1035.

13. Ghomi, M., The problem of optimal smoothing for convex functions, Proc. Amer. Math. Soc. 130 (2002), no. 8, 2255-2259.

14. Hanzawa, E., Classical solutions of the Stefan problem, Tohoku Math. J. (2), Volume 33, Number 3 (1981), 297-335.

15. Ishige, K., Salani, P., Is quasi-concavity preserved by heat flow?, Arch. Math. (Basel) 90 (2008), no. $5,450-460$.

16. Ishige, K., Salani, P., Convexity breaking of the free boundary for porous medium equations, Interfaces Free Bound. 12 (2010), no. 1, 75-84.

17. Ishige, K., Salani, P., On a new kind of convexity for solutions of parabolic problems, Discrete Contin. Dyn. Syst. Ser. S 4 (2011), no. 4, 851-864.

18. Kim, I.C. Uniqueness and existence results on the Hele-Shaw and the Stefan problems, Arch. Ration. Mech. Anal. 168 (2003), no. 4, 299-328.

19. Kamenomostskaja, S. L., On Stefan's problem, (Russian) Mat. Sb. (N.S.) 53 (95) 1961, 489-514.

20. Kinderlehrer, D., Nirenberg, L., The smoothness of the free boundary in the one phase Stefan problem, Comm. Pure Appl. Math. 31 (1978), no. 3, 257-282.

21. Meirmanov, A. M., The classical solution of a multidimensional Stefan problem for quasilinear parabolic equations, (Russian) Mat. Sb. (N.S.) 112(154) (1980), no. 2(6), 170-192.

22. Oleinik, O. A. A method of solution of the general Stefan problem, Dokl. Akad. Nauk SSSR 135 1054-1057 (Russian); translated as Soviet Math. Dokl. 1 (1960), 1350-1354.

23. Rubinstein, L. I., On the solution of Stefan's problem, (Russian) Bull. Acad. Sci. URSS. Sér. Géograph. Géophys. [Izvestia Akad. Nauk SSSR] 11, (1947). 37-54.

24. Rubinstein, L. I., The Stefan problem, Translated from the Russian by A. D. Solomon. Translations of Mathematical Monographs, Vol. 27. American Mathematical Society, Providence, R.I., 1971.

Department of Mathematics, The University of British Columbia, 1984 Mathematics Road, Vancouver, B.C., Canada V6T 1Z2. Email: Chau@math.ubc.ca.

Department of Mathematics, Northwestern University, 2033 Sheridan Road, Evanston, IL 60208, USA. EMAIL: WEINKOVE@MATH.NORTHWESTERN.EDU. 